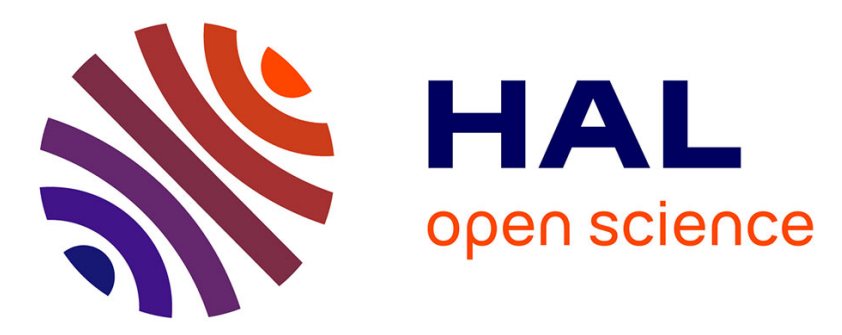

\title{
High-speed mobile robot control in off-road conditions: a multi-model based adaptive approach
}

\author{
R. Lenain, Benoît Thuilot, O. Hach, P. Martinet
}

\section{To cite this version:}

R. Lenain, Benoît Thuilot, O. Hach, P. Martinet. High-speed mobile robot control in off-road conditions: a multi-model based adaptive approach. IEEE International Conference on Robotics and Automation, ICRA'11, May 2011, Shangaï, China. p. 6143 - p. 6149. hal-00615483

\section{HAL Id: hal-00615483 \\ https://hal.science/hal-00615483}

Submitted on 19 Aug 2011

HAL is a multi-disciplinary open access archive for the deposit and dissemination of scientific research documents, whether they are published or not. The documents may come from teaching and research institutions in France or abroad, or from public or private research centers.
L'archive ouverte pluridisciplinaire HAL, est destinée au dépôt et à la diffusion de documents scientifiques de niveau recherche, publiés ou non, émanant des établissements d'enseignement et de recherche français ou étrangers, des laboratoires publics ou privés. 


\title{
High-speed mobile robot control in off-road conditions: a multi-model based adaptive approach
}

\author{
Roland Lenain ${ }^{1}$, Benoit Thuilot ${ }^{2,4}$, Oliver Hach ${ }^{1}$, Philippe Martinet ${ }^{3,4}$ \\ ${ }^{1}$ Cemagref, 24 avenue des Landais, 63172 Aubière, France \\ ${ }^{2}$ Clermont Université, Université Blaise Pascal, LASMEA, BP 10448, 63000 Clermont-Ferrand, France \\ ${ }^{3}$ Clermont Université, IFMA, LASMEA, BP 10448, 63000 Clermont-Ferrand, France $\quad{ }^{4}$ CNRS, UMR 6602, LASMEA, 63177 Aubière, France \\ roland.lenain@cemagref.fr \\ benoit.thuilot@lasmea.univ-bpclermont.fr
}

\begin{abstract}
This paper is focused on the design of a control strategy for the path tracking of off-road mobile robots acting at high speed. In order to achieve high accuracy in such a context, uncertain and fast dynamics have to be explicitly taken into account. Since these phenomena (grip conditions, delays due to inertial and low-level control properties) are hardly measurable directly, the proposed approach relies on predictive and observer-based adaptive control techniques. In particular, the adaptive part is based on an observer loop, taking advantage of both kinematic and dynamic vehicle models. This multimodel based adaptive approach permits to adapt on-line the grip conditions (represented by cornering stiffnesses), enabling highly reactive sideslip angles observation and then accurate path tracking. The relevance of this approach is investigated through full scale experiments.
\end{abstract}

\section{INTRODUCTION}

Off-road mobile robots are a subject of increasing popularity, since their potential applications may meet social needs, especially concerning environmental issues. From surveillance to agricultural operations, the use of robots in natural environment may permit to increase the work efficiency, while reducing pollution as well as the risk for human personnel [1]. In order to make such applications feasible, autonomous path tracking operations must demonstrate high accuracy, even at high speed (see for instance [2]). This implies the design of advanced control strategies, since classical control approaches [3] relying on the assumption of rolling without sliding are no longer valid in the off-road context, especially at high speed. Robust approaches have been investigated by considering sliding as a perturbation (see for instance [4] or [5]). Nevertheless, the efficiency of such approaches is limited, since they tend to be conservative, leading to an oscillating behavior of the robots even at limited speed.

Consequently, model-based control seems to be more attractive. However, simple kinematic approaches cannot reflect the entire robot behavior, while complete dynamic descriptions (as achieved in [6]) require the knowledge of numerous parameters. For example the ground-tire interaction model described in [7] employs 14 parameters, which are subject to changes in natural environment (as grip conditions

This work is supported by French National Research Agency (ANR), under the grant ANR-07-ROBO-0008 attributed to the FAST project, see https://projetfast.cemagref.fr/ are not constant). In [8], an adaptive approach has been proposed, based on both extended kinematic and dynamic models. The control law is designed from the extended kinematic model, consisting in a Ackermann model that additionally accounts for the sideslip angles. Then, based on dynamical equations of motion, an observer is designed to estimate the grip conditions and the sideslip angles needed in the control law expression. However, the matrix inversion required in grip condition estimation becomes singular when the robot does not laterally slide (for instance, in straight line motion). In this case, the estimation process must be stopped. Most of the time, there is no serious consequence, since the mobile robot does not slide. Nevertheless, such switches can generate delay and transient lacks of accuracy, and exceptionally instabilities can occur.

In this paper, a new strategy for grip condition estimation is proposed, relying on a sensitivity-based gradient search algorithm, and its integration into the adaptive control method is shown. In contrast to the method presented in [8], it allows a continuous estimation without any matrix inversion. Section II of this article pictures the modelling of an off-road mobile robot from kinematic and from dynamic points of view. Next, the control law, based on the extended kinematic model, is presented in Section III. The estimation of the sideslip angles, needed in the control law, is then described in Section IV. The estimation process is composed of several steps: first, a preliminary estimation of the sideslip angles relying on the extended kinematic model (slow-varying estimation), then the estimation of tire-soil interaction forces and the adaptation of the grip conditions (represented by cornering stiffnesses) and finally the observation of the sideslip angles based on the dynamic model (enabling a high reactive observation). In section $\mathrm{V}$, results of experiments in off-road conditions show the efficiency of the approach in terms of path tracking accuracy at relatively high speed (up to $6 \mathrm{~m} \mathrm{~s}^{-1}$ ).

\section{Mobile Robot Modelling}

\section{A. Scheme and notation}

As the work described in this paper is dedicated to off-road mobile robot control, the classical "rolling without sliding" assumption can no longer be applied (it would lead to large tracking errors). Thus, a purely kinematic model cannot 
be used in such a context. Two alternative representations are therefore defined below, using the common framework depicted in Fig. 1.

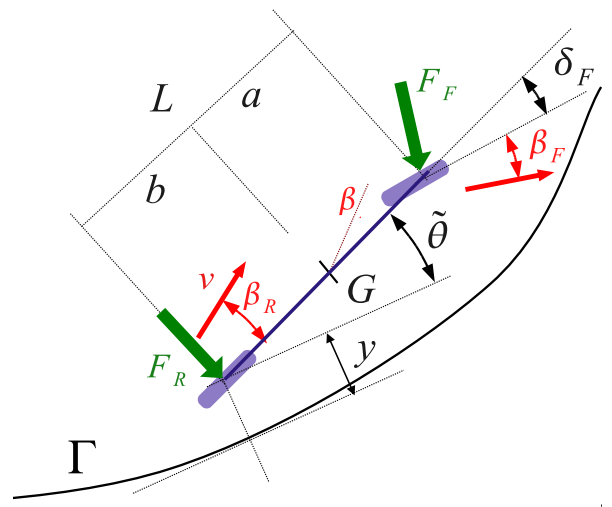

Fig. 1. Path tracking parameters

The mobile robot is considered as a bicycle, whose motion is controlled with respect to a reference path $\Gamma$. The common modelling variables and parameters are listed below:

- $L=a+b$ is the vehicle wheel base, where $a$ and $b$ are the distances of the front and rear wheel from the center of gravity $G$,

- $F_{F}$ and $F_{R}$ are the lateral forces generated at the front and rear tires, respectively,

- $m$ and $I_{z}$ are dynamic parameters (mass and inertia along the vertical axis),

- $v$ is the vehicle linear velocity at the center of the rear axle (point to be controlled),

- $\delta_{F}$ is the front steering angle, it constitutes the control variable,

- $\beta_{F}$ and $\beta_{R}$ are the front and rear side slip angles, while $\beta$ denotes the global sideslip angle,

- $c(s)$ is the curvature of the path $\Gamma$, parametrized by its arc-length coordinate $s$,

- $\theta$ is the vehicle orientation w.r.t. an absolute frame

- $\tilde{\theta}$ is the angular deviation of the vehicle w.r.t. $\Gamma$,

- $y$ is the vehicle lateral deviation w.r.t. $\Gamma$.

\section{B. Dynamic model}

In order to take into account skidding effects and other dynamic phenomena, the natural approach consists in considering a dynamical model of motion (i.e. linking forces and accelerations). When considering only the lateral behavior of the mobile robot, the following dynamical model is commonly proposed in the literature (see [6]):

$$
\left\{\begin{aligned}
\ddot{\theta} & =\frac{1}{I_{z}}\left(-a F_{F} \cos \left(\delta_{F}\right)+b F_{R}\right) \\
\dot{\beta} & =-\frac{1}{v_{2} m}\left(F_{F} \cos \left(\beta-\delta_{F}\right)+F_{R} \cos (\beta)\right)-\dot{\theta} \\
\beta_{R} & =\arctan \left(\tan \beta-\frac{b \dot{\theta}}{v_{2} \cos (\beta)}\right) \\
\beta_{F} & =\arctan \left(\tan \beta+\frac{a \dot{\theta}}{v_{2} \cos (\beta)}\right)-\delta_{F} \\
v_{2} & =v \frac{\cos \left(\beta_{R}\right)}{\cos (\beta)}
\end{aligned}\right.
$$

If lateral forces $F_{F}$ and $F_{R}$ are known, then system (1) accurately describes the robot dynamics. However, it does not rely directly on the controlled variable (lateral deviation $y$ ), and the required integrations make this model hardly tractable for control purposes.

\section{Extended kinematic model}

In order to design a relevant control law for high speed path tracking in off-road context, an alternative model is designed, comprising the lateral deviation as well as sliding effects. As introduced in [9] and generalized in [5], it is based on a kinematic description of the bicycle motion, to which the sideslip angles that appear in the dynamic model (1) are added. The motion of the robot can then be defined by the system of equations (2), which constitutes the extended kinematic model.

$$
\left\{\begin{aligned}
\dot{s} & =v \frac{\cos \left(\tilde{\theta}+\beta_{R}\right)}{1-c(s) y} \\
\dot{y} & =v \sin \left(\tilde{\theta}+\beta_{R}\right) \\
\dot{\tilde{\theta}} & =v\left[\cos \left(\beta_{R}\right) \lambda_{1}-\lambda_{2}\right]
\end{aligned}\right.
$$

with: $\lambda_{1}=\frac{\tan \left(\delta_{F}+\beta_{F}\right)-\tan \left(\beta_{R}\right)}{L}, \lambda_{2}=\frac{c(s) \cos \left(\tilde{\theta}+\beta_{R}\right)}{1-c(s) y}$

$s, y$ and $\tilde{\theta}$ are assumed to be measured by appropriate sensors (an RTK GPS receiver and a gyrometer). If $\beta_{F}$ and $\beta_{R}$ are also available (sliding observation is described in section IV), then model (2) can describe accurately the mobile robot motion, including sliding effects.

\section{CONTROL LAW}

The structure of model (2) remains close to a purely kinematic description, as reported in [10]. In particular, it preserves the exact linearization properties of these descriptions. This attractive feature can then be used to derive a nonlinear control law, considering the sliding variables $\beta_{F}$ and $\beta_{R}$ as known: as described in [11], once the exact linearization is achieved via nonlinear state and control transformations, a classical PD control can be proposed for the auxiliary inputs in order to ensure the convergence of the actual lateral deviation to zero. The reverse nonlinear transformations provide finally the nonlinear expression (3) for the steering control law.

$\delta_{F}=\arctan \left(\tan \left(\beta_{R}\right)+\frac{L}{\cos \left(\beta_{R}\right)}\left(\frac{c(s) \cos \tilde{\theta}_{1}}{\alpha}+\frac{A \cos ^{3} \tilde{\theta}_{1}}{\alpha^{2}}\right)\right)-\beta_{F}$

with $K_{p}$ and $K_{d}$ strictly positive tunable gains and:

$$
\left\{\begin{aligned}
\tilde{\theta}_{1} & =\tilde{\theta}+\beta_{R} \\
\alpha & =1-c(s) y \\
A & =-K_{p} y-K_{d} \alpha \tan \tilde{\theta}_{1}+c(s) \alpha \tan ^{2} \tilde{\theta}_{1}
\end{aligned}\right.
$$

In practice, steering actuators introduce some delay, which may damage the path tracking performances of control law (3). Consequently, Model Predictive Control techniques are here applied to address specifically curvature servoing. More precisely, the steering control law is split into two additive terms:

$$
\delta_{F}=\delta_{\text {Traj }}+\delta_{\text {Deviation }}
$$


$\delta_{\text {Deviation }}$ is a term mainly concerned with control errors and sliding compensation, while $\delta_{\operatorname{Traj}}$ deals with the reference path's shape: it imposes that the robot's orientation follows the curvature of the path. As the future curvature of the path to be followed is known, as well as the steering actuator features, a model predictive algorithm can be derived: the

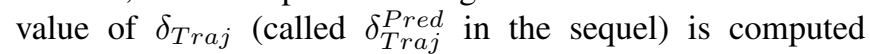
regarding an optimality criterion, in order to reach "at best" the future curvature on a fixed horizon of prediction. Substituting $\delta_{\text {Traj }}$ by this term, leads to the adaptive and predictive control law:

$$
\delta_{F}=\delta_{\text {Traj }}^{\text {Pred }}+\delta_{\text {Deviation }}
$$

\section{SIDESLIP ANGLE OBSERVATION}

Control law (6) requires indeed the knowledge of the sideslip angles $\beta_{F}$ and $\beta_{R}$. As they are hardly measurable directly, their indirect estimation is addressed here. It is based on a algorithm, which relies on both kinematic and dynamic vehicle models, thus enabling accurate control even at high speed. The different steps of the hierarchical estimation process are detailed below.

\section{A. Sideslip angle estimation from extended kinematic model}

When considering kinematic model (2) from an observation point of view, it can be noticed that it is composed of two equations (the $\dot{s}$ equation is indeed of no use, since only lateral motion is here considered), when only two unknowns have to be estimated $\left(\beta_{F}, \beta_{R}\right)$. Such an observation problem can be solved as described in detail in [11]. The extended kinematic model (2) can be rewritten as:

$$
\begin{aligned}
\dot{X}_{o b s} & =f\left(X_{o b s}, \delta_{F}, u\right) \\
& =\left\{\begin{array}{l}
v \sin \left(\tilde{\theta}_{o b s}+u_{R}\right) \\
v\left[\frac{\cos \left(u_{R}\right)\left[\tan \left(\delta_{F}+u_{F}\right)-\tan \left(u_{R}\right)\right]}{L}-\frac{c(s) \cos \left(\tilde{\theta}_{o b s}+u_{R}\right)}{1-c(s) y_{o b s}}\right]
\end{array}\right.
\end{aligned}
$$

where $X_{o b s}=\left[\begin{array}{ll}y_{o b s} & \tilde{\theta}_{o b s}\end{array}\right]^{T}$ is the observed state vector and $u=\left[\begin{array}{ll}u_{F} & u_{R}\end{array}\right]^{T}=\left[\begin{array}{ll}\beta_{F} & \beta_{R}\end{array}\right]^{T}$ are the sideslip angles to be estimated. The general principle is here to consider the sideslip angles $u$ as control variables to be designed in order to achieve the convergence of $X_{o b s}$ to the measured state $X_{m e s}=\left[\begin{array}{ll}y_{m e s} & \tilde{\theta}_{m e s}\end{array}\right]^{T}$.

As sideslip angles do not exceed a few degrees in practice, this state equation can be linearized with respect to the control vector $u$ in the vicinity of zero (i.e. no sliding), leading to

$$
\dot{X}_{o b s}=f\left(X_{o b s}, \delta_{F}, 0\right)+B\left(X_{o b s}, \delta_{F}\right) u
$$

with $B(.,$.$) denoting the derivative of f$ with respect to $u$, evaluated at $u=(0,0)$.

Provided that $\tilde{\theta}_{o b s} \neq \frac{\pi}{2}[\pi]$ and $v \neq 0$, the matrix $B$ is invertible. Let $e=X_{o b s}-X_{m e s}$ be the observed error. Then, an exponential convergence $\dot{e}=G e$ can be obtained by choosing:

$$
u=B\left(X_{o b s}, \delta_{F}\right)^{-1}\left(G e+\dot{X}_{m e s}-f\left(X_{o b s}, \delta_{F}, 0\right)\right)
$$

$G$ has to be chosen as a Hurwitz matrix and constitutes the observer gain, assigning the settling times for the state observation. Since convergence of the observed state $X_{\text {obs }}$ to the measured one $X_{m e s}$ is achieved, $u$ can be regarded as a relevant estimate of the sideslip angles. This first observation step supplies a satisfactory sliding estimate in off-road context at limited speed: the results reported for instance in [11] show very accurate results up to $4 \mathrm{~m} \mathrm{~s}^{-1}$. Nevertheless, since this observer is designed from a kinematic model, the observation of dynamical variables such as sideslip angles necessarily presents some delays, that may generate oscillations when the robot's velocity is increased.

\section{B. Dynamic sideslip angle estimation}

In order to improve the reactivity of the sideslip angle estimation, the design of an observer based on dynamic model (1) is preferable. In order to use such a model, an analytical expression of the two forces $F_{F}$ and $F_{R}$ is required. A linear expression, quite standard in the literature (see for instance [6]), is here considered:

$$
\left\{\begin{array}{l}
F_{F}=C_{F} \beta_{F} \\
F_{R}=C_{R} \beta_{R}
\end{array}\right.
$$

where $C_{F}$ and $C_{R}$ are the cornering stiffnesses. While such a linear expression can be valid in on-road context, the motion in natural environment (moreover at high speed) implies non-linear tire-ground interaction forces. Nevertheless, as demonstrated in [8], the linear model (10) can still be used, provided that cornering stiffnesses are on-line adapted, in order to be representative of the non-linearity and the variability of the grip conditions.

On the condition that $C_{F}$ and $C_{R}$ can be properly online adapted, the integration of the tire forces (10) into the dynamic model (1) leads to the following state space representation (the third and fourth equations in (1) have been used to express $\beta_{F}$ and $\beta_{R}$ as functions of $\beta$ ):

$$
\dot{X}_{2}=A_{2} X_{2}+B_{2} \delta_{F}
$$

where:

$$
A_{2}=\left[\begin{array}{cc}
\frac{-a^{2} C_{F}-b^{2} C_{R}}{v_{2} I_{z}} & \frac{-a C_{F}+b C_{R}}{I_{z}} \\
-\frac{a C_{F}-b C_{R}}{v_{2}^{2} m}-1 & -\frac{C_{F}+C_{R}}{v_{2} m}
\end{array}\right], \quad B_{2}=\left[\begin{array}{c}
\frac{a C_{F}}{I_{F}} \\
\frac{C_{F}}{v_{2} m}
\end{array}\right]
$$

$X_{2}=\left[\begin{array}{ll}\dot{\theta} & \beta\end{array}\right]^{T}$ is the state vector, $\delta_{F}$ is the control variable. Since the steering anlge is limited to $20^{\circ}$, it has been assumed that $\cos \delta_{F} \approx 1$. This allows to simplify the matrix $A_{2}$ and turns the dynamic model (1) into the linear form (12).

It has also been assumed that the first state variable $\dot{\theta}$ can be measured (for instance, with a gyrometer). An estimation of the second state variable $\beta$ can be inferred from (9). Let us denote this estimation as $\bar{\beta}$ and let us consider $\bar{\beta}$ as a virtual measurement of $\beta$. Then, a standard observer equation associated with model (11) is:

$$
\dot{\hat{X}}_{2}=A_{2} \hat{X}_{2}+B_{2} \delta_{F}+G_{2} \tilde{X}_{2}
$$

where $\hat{X}_{2}=\left[\begin{array}{ll}\dot{\hat{\theta}}_{2} & \hat{\beta}_{2}\end{array}\right]^{T}$ is the observed state, $\bar{X}=\left[\begin{array}{ll}\dot{\bar{\theta}} & \bar{\beta}\end{array}\right]^{T}$ is the measured state and $\tilde{X}_{2}=\hat{X}_{2}-\bar{X}$ is the observer error. 
From (11) and (13), it can be deduced that:

$$
\dot{\tilde{X}}_{2}=\left(A_{2}+G_{2}\right) \tilde{X}_{2}
$$

The convergence of the observer error $\tilde{X}_{2}$ to zero is then ensured, provided that $G_{2}$ is chosen such that $A_{2}+G_{2}$ is Hurwitz. $\bar{\beta}$ has been chosen as the measurement associated with the observed state $\hat{\beta}_{2}$, since its steady state value is always correct. However, since the values of $\bar{\beta}$ are not necessarily accurate during transient phases, the preference is given to the convergence of $\dot{\hat{\theta}}_{2}$ (since yaw rate measurement is reliable) with respect to the convergence of $\hat{\beta}_{2}$. This can easily be achieved by tuning $G_{2}$ such that the settling time associated with $\dot{\hat{\theta}}_{2}$ is shorter than the one associated with $\hat{\beta}_{2}$.

Finally, the front and rear sideslip angles to be injected into control law (6) can be obtained by reporting $\hat{\beta}_{2}$ into the third and fourth equations of (1):

$$
\left\{\begin{array}{l}
\hat{\beta}_{R}=\hat{\beta}_{2}-\frac{b \dot{\hat{\theta}}_{2}}{v_{2}} \\
\hat{\beta}_{F}=\hat{\beta}_{2}+\frac{a \hat{\hat{\theta}}_{2}}{v_{2}}-\delta_{F}
\end{array}\right.
$$

Equations (15) can be interpreted as a mixed kinematic and dynamic sideslip angle observer. As demonstrated in Section V, observer (15) improves the robustness and the reactivity of the sideslip angle estimation at high speed with respect to observer (9), and thus the performances of path tracking control.

\section{Tire force observation and cornering stiffness adaptation}

The previous observer indeed relies on relevantly adapted cornering stiffnesses $C_{F}$ and $C_{R}$. When considering the dynamical model (1), together with the expression (10) for tire forces, the adaptation of the cornering stiffnesses can be achieved in the same way as for observer (9). Such an approach (named "Direct Adaptation" in the sequel) has been presented in [8]. However, it requires the inversion of a matrix, which becomes singular in the non-sliding case (i.e. when $\beta=0$ ). This situation is likely to occur, even in off-road context (for instance, when following straight line path segments), and in such cases the adaptation must be stopped. Most of the time, these transient inaccuracies in cornering stiffness estimation have no serious consequence, since sideslip angles, and therefore lateral forces, are small. However, this switching behaviour can nevertheless generate delay and transient lacks of accuracy when the mobile robot leaves the straight line and enters into a curve, and, at worst, instabilities can occur.

This problem is addressed here by using the MIT Rule (as presented in [12]), considering the cornering stiffnesses as slow-varying parameters of force model (10). The first step is the estimation of the forces $F_{F}$ and $F_{R}$. It is achieved by rewriting model (1) as follows (considering that the values of $\beta$ are small):

$$
\dot{\hat{X}}_{c}=A_{c} \hat{X}_{c}+B_{c}\left(\delta_{F}\right)\left[\begin{array}{ll}
\hat{F}_{F} & \hat{F}_{R}
\end{array}\right]^{T}
$$

where:

$$
A_{c}=\left[\begin{array}{cc}
0 & 0 \\
-1 & 0
\end{array}\right], \quad B_{c}\left(\delta_{F}\right)=\left[\begin{array}{cc}
-\frac{a \cos \delta_{F}}{I_{z}} & \frac{b}{I_{z}} \\
-\frac{\cos \delta_{F}}{v_{2} m} & -\frac{1}{v_{2} m}
\end{array}\right]
$$

$\hat{X}_{c}=\left[\begin{array}{ll}\dot{\hat{\theta}}_{c} & \hat{\beta}_{c}\end{array}\right]^{T}$ is the observed state, $\left[\begin{array}{ll}\hat{F}_{F} & \hat{F}_{R}\end{array}\right]^{T}$ are the forces to be estimated.

The vector $\bar{X}$, introduced in section IV.B, can again be seen as a measurement of $\hat{X}_{c}$. Then, the tire forces can be estimated by imposing the convergence of $\hat{X}_{c}$ to $\bar{X}$. The matrix $B_{c}\left(\delta_{F}\right)$ being invertible as soon as the velocity $v_{2}$ is non-zero, this convergence can be obtained by:

$$
\left[\begin{array}{ll}
\hat{F}_{F} & \hat{F}_{R}
\end{array}\right]^{T}=B_{c}\left(\delta_{F}\right)^{-1}\left(G_{c} \tilde{X}_{c}-A_{c} \hat{X}_{c}\right),
$$

where $\tilde{X}_{c}=\hat{X}_{c}-\bar{X}$ and $G_{c}$ is a gain matrix. The expression (18) indeed leads to the following error dynamics, provided that $\dot{\bar{X}}$ is negligible:

$$
\dot{\tilde{X}}_{c}=G_{c} \tilde{X}_{c}
$$

The estimates $\hat{F}_{i}{ }_{i \in\{F, R\}}$ can then be considered as the actual lateral tire forces applied to the robot. Consequently, in view of (10), and taking into account that cornering stiffnesses are slow-varying with respect to other dynamics, a relevant estimation $\hat{C}_{i}$ has to satisfy:

$$
\hat{F}_{i}=\hat{C}_{i} u_{i}
$$

where $u_{i}$ are the sideslip angles estimated by (9).

Relying on a sensitivity-based gradient search algorithm, a cornering stiffness adaptation law is then:

$$
\dot{\hat{C}}_{i}=-\gamma\left(\hat{F}_{i}-\hat{C}_{i} u_{i}\right) \frac{\partial\left(\hat{F}_{i}-\hat{C}_{i} u_{i}\right)}{\partial \hat{C}_{i}}=-\gamma\left(\hat{F}_{i}-\hat{C}_{i} u_{i}\right) u_{i}
$$

where $\gamma$ is a strictly positive tunable gain.

Adaptation law (21) (named "MIT Adaptation" in the sequel) thus permits to adapt the cornering stiffnesses $\hat{C}_{i \in\{F, R\}}$, so that model (10) is consistent with both the estimation of the tire forces and the kinematic estimation of the sideslip angles. The dynamic observer (15) can then be run and supply the reactive estimates of the sideslip angles needed in control law (6).

\section{Summary of the observer approach}

The hierarchical observer proposed in this paper and composed of the previously described steps can be summarized by the scheme in Fig. 2 .

The first step, shown as a red/dashed box in Fig. 2, consists in the preliminary observer (9), derived from the extended kinematic model (2). It supplies a first estimation of the sideslip angles, considered accurate enough to estimate slow-varying parameters. This estimation, considered as a virtual measurement, is then used together with the measured yaw rate in order to estimate lateral forces: it consists in observer (18), derived from dynamical model (1). In turn, from these slow-varying estimates of the forces and of the sideslip angles, the cornering stiffnesses (also considered as slow-varying) are adapted via the expression (21), derived 


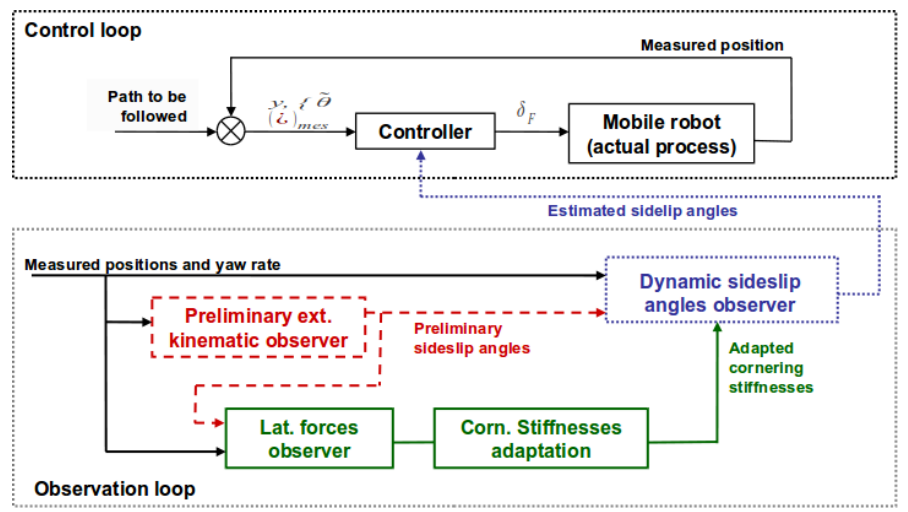

Fig. 2. Observer principle scheme

also from dynamical model (1). This second step is shown as green/plain boxes in Fig. 2. All the parameters of dynamical model (1) are then known (mass and inertial parameters are invariant, and cornering stiffnesses are supplied by expression (21)), so that a second sideslip angle observer, more reactive since it takes explicitly into account dynamics effects, can be derived: it consists in equations (13) and (15) and is shown as a blue/dashed box in Fig. 2. Finally, this last estimation of the sideslip angles is entered into the adaptive and predictive control law (6), in order to achieve accurate path tracking.

\section{EXPERIMENTAL RESULTS}

\section{A. Experimental setup}

The electric off-road vehicle depicted in Fig. 3 is used as an experimental platform. Designed for all-terrain mobility, it can climb slopes up to $45^{\circ}$, its maximum speed is $8 \mathrm{~m} \mathrm{~s}^{-1}$, and it has the following properties:

\section{TABLE I}

EXPERIMENTAL ROBOT DYNAMIC PARAMETERS

$\begin{array}{cc}\text { Total mass } & m=350 \mathrm{~kg} \\ \text { Yaw inertia } & I_{z}=270 \mathrm{~kg} \mathrm{~m}^{2} \\ \text { Wheelbase } & L=1.2 \mathrm{~m} \\ \text { Rear half-wheelbase } & b=0.58 \mathrm{~m}\end{array}$

The main exteroceptive sensor on-board is a "Magellan ProFlex 500" RTK-GPS receiver, which supplies absolute position measurements with an accuracy of $2 \mathrm{~cm}$ at a $10 \mathrm{~Hz}$ sampling frequency. The GPS antenna is located vertically above the center of the rear axle, so that the absolute position of the point to be controlled is straightforwardly obtained from the sensor. In addition, a MTi IMU manufactured by Xsens is on-boarded. In this framework, only the vertical axis of the gyrometer, supplying the yaw rate is used. It supplies this measure with an accuracy of $0.1 \% \mathrm{~s}$ and is fixed on the chassis.

\section{B. Path tracking results}

The results presented in this paper are illustrated by an extended video, available at ftp://ftp.clermont.cemagref.fr/pub/Tscf/Lenain/VideoIcra2011/

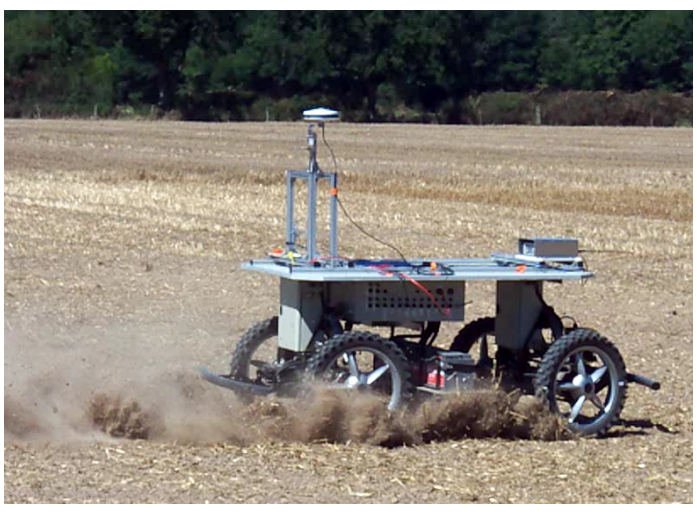

Fig. 3. Experimental platform

1) Relevance of cornering stiffness adaptation: The first experiment for the algorithm's validation consists in following the path depicted in Fig. 4. This path has been recorded beforehand, when the robot was steered manually at $1 \mathrm{~m} \mathrm{~s}^{-1}$. It is composed of two circles: one performed on asphalt and the other one performed on dry grass.

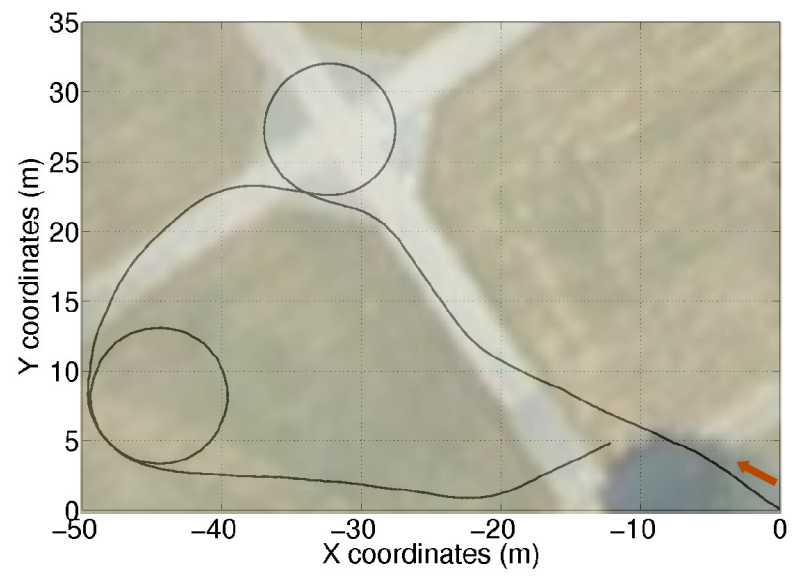

Fig. 4. Reference trajectory manually recorded

This trajectory has been followed twice, at $4 \mathrm{~m} \mathrm{~s}^{-1}$, using control law (6) fed by observer (15):

- In the first run, the cornering stiffnesses are estimated via the "Direct Adaptation" method described in [8]. The results are reported in red dotted line. Switches in the adaptation of the cornering stiffnesses, due to a matrix singularity (see discussion in Section IV.C), are encountered.

- In the second run, the cornering stiffness adaptation is ensured by the "MIT Adaptation" approach (i.e. according to (21)). The results are reported in magenta solid line.

Fig. 5 shows a comparison plot of the resulting tracking errors. It can first be noticed that both adaptation strategies allow accurate tracking: after initialization (i.e. after $5 \mathrm{~s}$ ) the lateral deviation does not exceed $50 \mathrm{~cm}$, while large oscillations with a constant deviation of more than $0.5 \mathrm{~m}$ during the circles are recorded when using a classical control 
law (i.e neglecting sliding, i.e. $\beta_{F}$ and $\beta_{R}$ are set to 0 ).

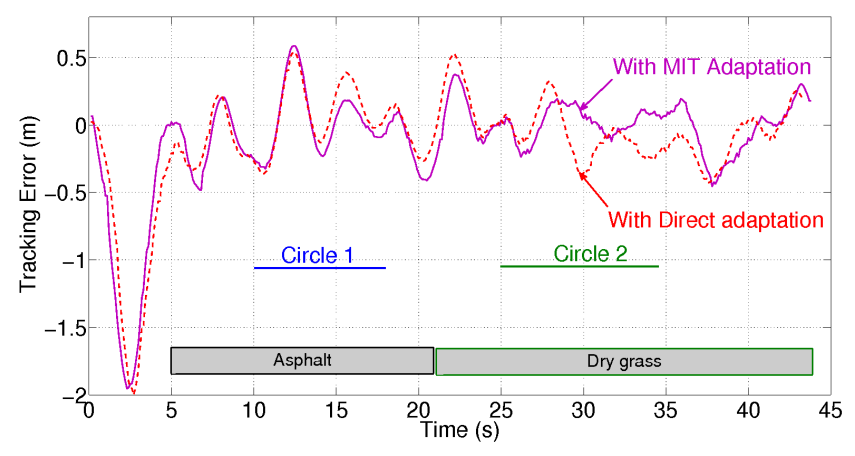

Fig. 5. Comparison of the tracking error at $4 \mathrm{~m} \mathrm{~s}^{-1}$

Nevertheless, some significant differences can be observed. First, it can be noticed that transient deviations in the tracking error are satisfactorily less important when the "MIT Adaptation" is used. The main difference can be seen during the second circle (from $25 \mathrm{~s}$ to $35 \mathrm{~s}$ ), as the error with the "MIT Adaptation" remains very close to zero, while the error with "Direct Adaptation" oscillates and converges to $-25 \mathrm{~cm}$. The explanation for this difference can be found by inspecting the recorded cornering stiffnesses. For the sake of clarity, only front cornering stiffnesses $C_{F}$ are compared in Fig. 6.

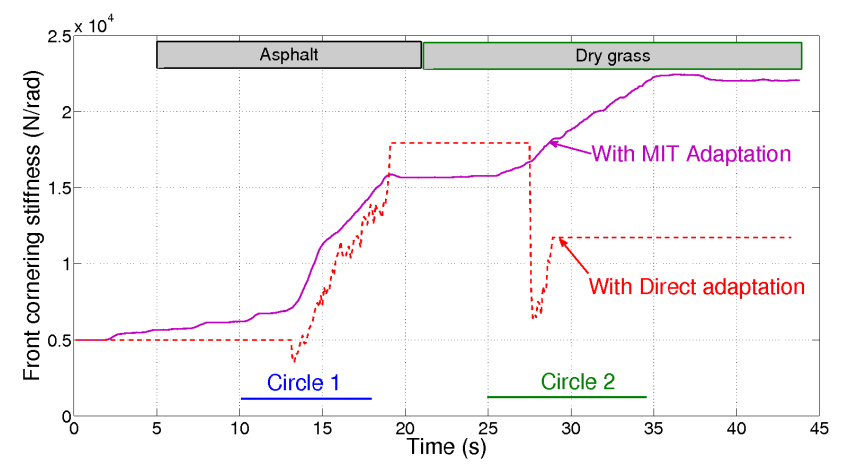

Fig. 6. Comparison of the adapted cornering stiffness

Obviously, the estimation obtained with the "MIT Adaptation" is smoother than with the "Direct Adaptation", and moreover no delay is added. It thus permits, relying on (15), a smooth sideslip angle estimation, leading to a more stable behavior in path tracking control. Indeed, the major improvement of the proposed observer with respect to "Direct Adaptation" is its continuous operation: even during straight line path segments (from $5 \mathrm{~s}$ to $10 \mathrm{~s}$ and from $17.5 \mathrm{~s}$ to $25 \mathrm{~s}$ ), the "MIT Adaptation" leads naturally to constant cornering stiffnesses (the estimated forces $\hat{F}_{i}$ and the sideslip angles $u_{i}$ are zero, leading to $\dot{\hat{C}}_{i}=0$ in equation (21)). On the contrary, such situations lead to singularities in the "Direct Adaptation", so that the adaptation process has to be stopped (before $12.5 \mathrm{~s}$, from $19 \mathrm{~s}$ to $27 \mathrm{~s}$ and after $29 \mathrm{~s}$ ). The cornering stiffnesses are then no longer adapted and stay at an arbitrary value, not necessarily representative of the actual grip conditions. As a result, an inaccurate estimation of the sideslip angles may be sent to the control law, explaining the large tracking errors recorded during the second circle.

This fact is highlighted in Fig. 7 that depicts a comparison of the front sideslip angles estimated via "Direct Adaptation" and via "MIT Adaptation", respectively. Differences in the cornering stiffness adaptation have indeed a direct impact on the sideslip angle estimation and consequently on the lateral deviation. They can be observed during both circular segments of the trajectory. During the first circle (10 s to $18 \mathrm{~s}$ ), the cornering stiffness adapted with "Direct Adaptation" starts varying after the one adapted with "MIT Adaptation", leading to an overestimation of the front sideslip angles (especially at the beginning of the circle at $12.5 \mathrm{~s}$ where a difference of $5^{\circ}$ is recorded). During the second circle (from $25 \mathrm{~s}$ to $35 \mathrm{~s}$ ), the "Direct Adaptation" method is interrupted because of a matrix singularity and the adapted cornering stiffness stays equal to $12500 \mathrm{~N} \mathrm{rad}^{-1}$. As a result, the corresponding estimation of the front sideslip angle is overestimated again (around $5^{\circ}$ instead of $2.5^{\circ}$ for the "MIT Adaptation").

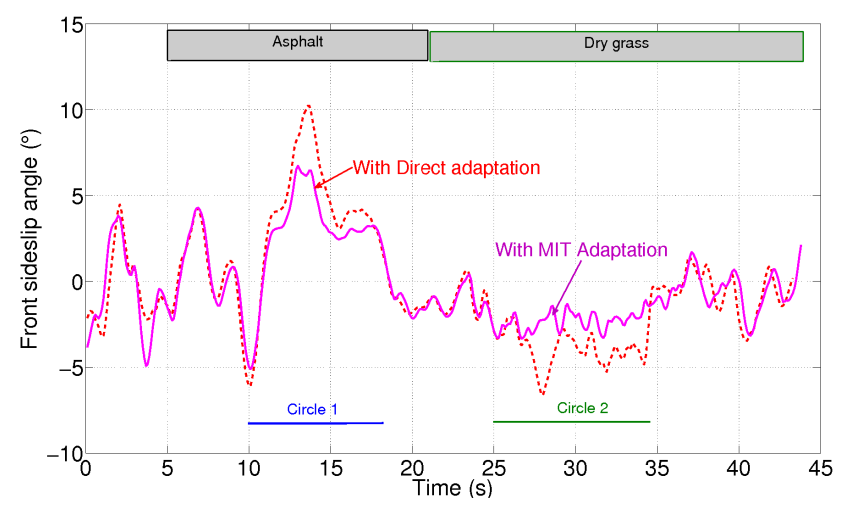

Fig. 7. Comparison of the estimated front sideslip angles

These differences in sideslip angle estimation also explain the disparities in the tracking error depicted in Fig. 5 and already commented. At worst, in other situations, the "Direct Adaptation" algorithm may even lead to instability, as it generates discontinuities in the adapted cornering stiffnesses (e.g. in Fig. 6 at 27 s). The proposed "MIT Adaptation" process is thereby more robust, due to its continuous operation.

2) High-speed validation: The above results permit to investigate path tracking at higher speed, close to the controllability limits of the robot. A second reference trajectory, still manually recorded at $1 \mathrm{~m} \mathrm{~s}^{-1}$, has been defined on the field shown in Fig. 3 and in the attached video. This trajectory is composed of a straight line, then a large circle and finally a quick inversion of the curvature (see Fig. 8).

Path tracking has then been achieved at $6 \mathrm{~m} \mathrm{~s}^{-1}$, using control law (6), observer (15) and "MIT Adaptation". The resulting path is displayed as dashed line in Fig. 8, while Fig. 9 shows the recorded tracking error.

It can be noticed that the maximal tracking error of $1 \mathrm{~m}$ is recorded in the beginning of the curve (around $12.5 \mathrm{~s}$ ), 


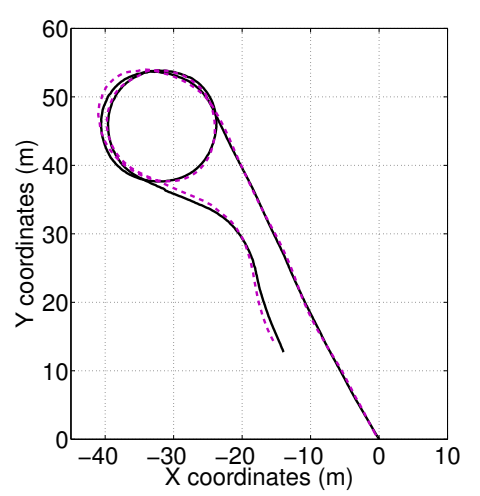

Fig. 8. Tracked path

while the lateral deviation afterwards converges to zero during the circle. A second transient overshoot occurs at $27 \mathrm{~s}$, corresponding to the small right curve (implying a fast curvature inversion). The large transient error is the result of two cumulative delays: first, the settling time of the steering actuator is $0.4 \mathrm{~s}$, meaning a settling distance of $2.4 \mathrm{~m}$ at the considered speed. Secondly, due to harsh grip conditions, variations in cornering stiffnesses are also reflected after a short delay: it can indeed be noticed, when examining the recorded cornering stiffnesses in Fig. 10, that at the beginning of the circle (around $12 \mathrm{~s}$ ) both $\hat{C}_{F}$ and $\hat{C}_{R}$, need $2 \mathrm{~s}$ to converge to nearly constant values, namely $5000 \mathrm{~N} \mathrm{rad}^{-1}$ and $4000 \mathrm{~N} \mathrm{rad}^{-1}$. This corresponds exactly to the duration of the transient deviation (from $12 \mathrm{~s}$ to $15 \mathrm{~s}$ ).

Therefore, in this experiment, in view of the actuator capabilities and the grip conditions, the mobile robot was close to its controllability limits, but nevertheless achieves successfully the path tracking mission. For slightly higher speed, this trajectory turns out to be non-admissible for this robot: at $7 \mathrm{~m} \mathrm{~s}^{-1}$, the robot starts spinning and is thus no longer able to follow the reference path.

\section{CONCLUSION AND FUTURE WORK}

This paper proposes a predictive and adaptive control law, dedicated to off-road path tracking of fast mobile robots. It is based on an observer scheme, mixing kinematic and dynamic models of the considered mobile robot. The

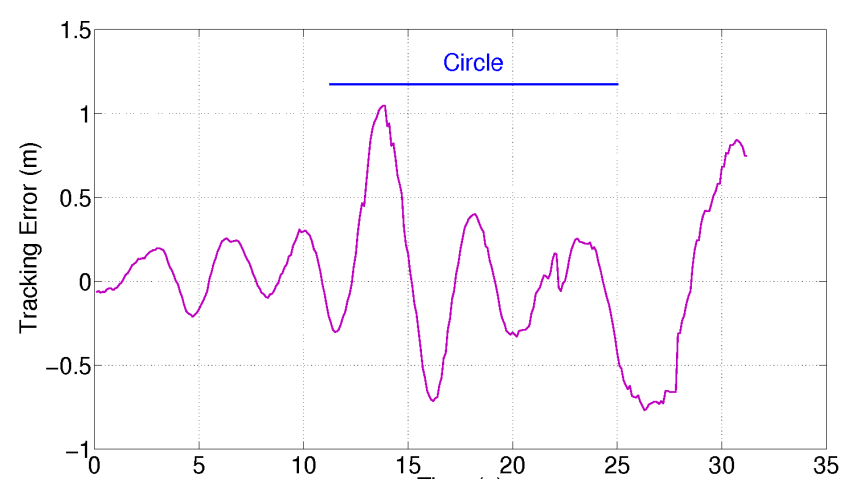

Fig. 9. Tracking error

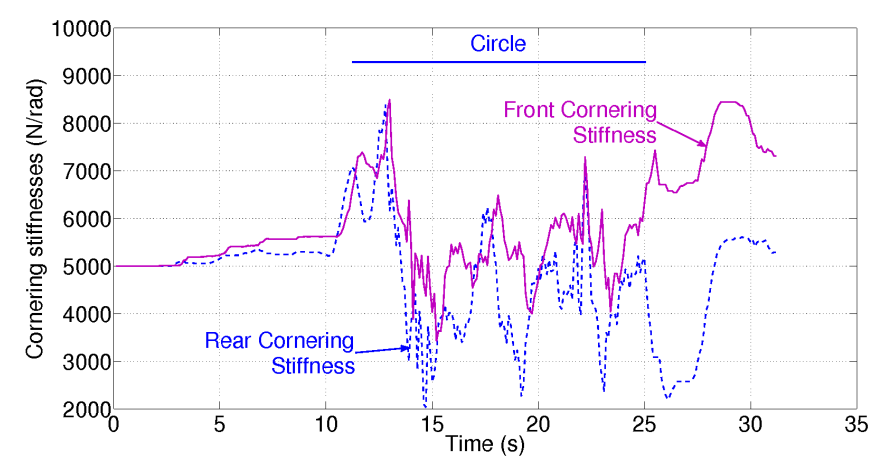

Fig. 10. Comparison of the adapted cornering stiffnesses

interaction between several levels of modelling allows the on-line estimation of the unknown dynamic model parameters, and finally the achievement of a reactive and relevant estimation of the sideslip angles. The observed variables are subsequently used in an associated control law, permitting highly accurate path tracking with respect to the considered speeds and grip conditions variabilities.

However, at high speed, reference paths may become nonadmissible, depending on the grip conditions. Current work is then focused on controlling the robot speed, in order to ensure that the reference path is always achievable, with respect to robot capabilities and grip conditions. This will permit to ensure a minimal error and to avoid spinning situations.

\section{REFERENCES}

[1] R. Siegwart and I. R. Nourbakhsh, Introduction to Autonomous Mobile Robots. MIT Press, 2004.

[2] H. Schäfer, M. Proetzsch, T. Braun, J. Koch, N. Schmitz, and K. Berns, "Ravon - a robust autonomous vehicle for off-road navigation," in European Land Robot Trial - ELROB, 2006.

[3] A. Micaelli and C. Samson, "Trajectory tracking for unicycle-type and two-steering-wheels mobile robots," INRIA, Tech. Rep. 2097, 1993.

[4] R. Eaton, J. Katupitiya, K. W. Siew, and K. S. Dang, "Precision guidance of agricultural tractors for autonomous farming," in Proc. 2nd Annual IEEE Systems Conference, 2008, pp. 1-8.

[5] D. Wang and C. Low, "An analysis of wheeled mobile robots in the presence of skidding and slipping: Control design perspective," in Proc. ICRA, 2007, pp. 2379-2384.

[6] M. Ellouze and B. d. Andréa-Novel, "Control of unicycle-type robots in the presence of sliding effects with only absolute longitudinal and yaw velocities measurement." European Journal of Control, vol. 6, pp. $567-584,2000$.

[7] E. Bakker, L. Nyborg, and H. Pacejka, "Tyre modeling for use in vehicle dynamics studies," International congress of Society of Automotive Engineers, Warrendale, Pensylvania, USA, pp. 2190-2204, 1987.

[8] R. Lenain, B. Thuilot, C. Cariou, and P. Martinet, "Multi-model based sideslip angle observer: Accurate control of high-speed mobile robots in off-road conditions," in Proc. IROS, 2009, pp. 1197-1202.

[9] —_, "Sideslip angles observers for vehicle guidance in sliding conditions: application to agricultural path tracking tasks," in Proc. ICRA, 2006, pp. 3183-3158.

[10] C. Samson, "Control of chained systems. Application to path following and time-varying point stabilization of mobile robots," IEEE Trans. Automat. Contr., vol. 40, pp. 64-77, 1995.

[11] C. Cariou, R. Lenain, B. Thuilot, and M. Berducat, "Automatic guidance of a four-wheel-steering mobile robot for accurate field operations," Journal of Field Robotics, vol. 26, pp. 504-518, 2009.

[12] K. Astrom and B. Wittenmark, Adaptive control (2nd edition). New York: Addison-Wesley, 1994. 